\title{
COINCIDENCE INVARIANTS AND HIGHER REIDEMEISTER TRACES
}

\author{
KATE PONTO
}

\begin{abstract}
The Lefschetz number and fixed point index can be thought of as two different descriptions of the same invariant. The Lefschetz number is algebraic and defined using homology. The index is defined more directly from the topology and is a stable homotopy class. Both the Lefschetz number and index admit generalizations to coincidences and the comparison of these invariants retains its central role. In this paper we show that the identification of the Lefschetz number and index using formal properties of the symmetric monoidal trace extends to coincidence invariants. This perspective on the coincidence index and Lefschetz number also suggests difficulties for generalizations to a coincidence Reidemeister trace.
\end{abstract}

\section{INTRODUCTION}

A coincidence point for a pair of maps $f, g: M \rightarrow N$ is a point $x$ of $M$ such that $f(x)=g(x)$. Coincidence points are a natural generalization of fixed points and there is a corresponding generalization of the Lefschetz fixed point theorem.

Theorem A. [13] Suppose $M$ and $N$ are closed, smooth, $\mathbb{Q}$-orientable manifolds of the same dimension and $f, g: M \rightarrow N$ are continuous maps. If $f$ and $g$ have no coincidence points then the Lefschetz number of $f$ and $g$

$L(f, g):=\sum_{i}(-1)^{i} \operatorname{tr}\left(\begin{array}{cc}H_{i}(M ; \mathbb{Q}) \stackrel{f_{*}}{\longrightarrow} H_{i}(N ; \mathbb{Q}) & H_{i}(M ; \mathbb{Q}) \\ -\left.\cap[N]\right|^{\operatorname{dim}(N)-i}(N ; \mathbb{Q}) \stackrel{g^{*}}{\rightarrow} H^{\operatorname{dim}(N)-i}(M ; \mathbb{Q})\end{array}\right)$

is zero.

The vertical maps above are the Poincaré duality isomorphism and they play in essential role in the definition of $L(f, g)$. The main result of this note is to give a simple proof of the following generalization.

Theorem B. Suppose $M$ and $N$ are closed, smooth manifolds and

$$
\theta: T \nu_{\triangle \subset N \times N} \wedge K \rightarrow L \wedge M_{+}
$$

Date: September 18, 2018.

719 Patterson Office Tower, Department of Mathematics, University of Kentucky, Lexington, KY, kate.ponto@uky.edu

The author was partially supported by NSF grant DMS-1207670. 
is a stable map for spaces (or spectra) $K$ and $L$. If continuous maps $f, g: M \rightarrow N$ have no coincidence points then

$\sum_{i}(-1)^{i} \operatorname{tr}\left(\tilde{H}_{i}\left(M_{+} \wedge K ; \mathbb{Q}\right) \stackrel{(f \times g)_{*}}{\longrightarrow} \tilde{H}_{i}\left(T \nu_{\triangle \subset N \times N} \wedge K ; \mathbb{Q}\right) \stackrel{\theta_{*}}{\longrightarrow} \tilde{H}_{i}\left(L \wedge M_{+} ; \mathbb{Q}\right)\right)$

is zero.

While the formulation of this result using the map $\theta$ is nonstandard, Theorem A and the generalizations in 20,21 follow from this result. It will allow us to prove a generalization of Theorem A where fundamental classes are replaced by arbitrary homology classes. See $\$ 2$,

The proofs here use duality and trace in symmetric monoidal categories [3, 17 . This allows for short, conceptual proofs that are very similar to the corresponding proofs of the Lefschetz fixed point theorem [3] and Reidemeister trace [16].

We finish by considering a generalization of the Lefschetz fixed point theorem for coincidences to Reidemeister traces. The approach here does not appear to generalize to this case and this failure is very suggestive. If there are algebraic generalizations of the Reidemeister trace to coincidences similar to the original definition in [7] we would expect to see an easy comparison of this invariant and a topological invariant using functoriality as for the Lefschetz number. Instead we have a very natural description of the Reidemeister trace for fixed points in terms of the categorical trace while the generalization to coincidences suggested by [1, 9 , is fundamentally incompatible with the trace.

Remark. In this paper we focus on closed smooth manifolds. Many of the results could also be stated in terms of compact ENRs (or finite CW complexes) by replacing normal bundles by mapping cylinders.

\section{TRACES For Symmetric MONOIDAL CATEGORIES}

The trace in symmetric monoidal categories is a generalization of the trace in linear algebra that retains many of the important properties. In particular, it satisfies a generalization of invariance of basis and is functorial. The generalized trace is a trace for endomorphisms of modules over a commutative ring, endomorphisms of chain complexes of modules over a commutative ring, and endomorphisms of closed smooth manifolds or compact ENRs. This section is a summary of [3, 14, 17.

Let $\mathscr{V}$ be a symmetric monoidal category with monoidal product $\otimes$, unit $S$, and symmetry isomorphism $\gamma$.

Definition 1.1. An object $A$ in $\mathscr{V}$ is dualizable with dual $A^{\star}$ if there are morphisms

$$
\eta: S \rightarrow A \otimes A^{\star} \text { and } \epsilon: A^{\star} \otimes A \rightarrow S
$$

such that the composites

$$
\begin{aligned}
A & \cong S \otimes A \stackrel{\eta \otimes \mathrm{id}}{\longrightarrow} A \otimes A^{\star} \otimes A \stackrel{\mathrm{id} \otimes \epsilon}{\longrightarrow} A \otimes S \cong A \\
A^{\star} & \cong A^{\star} \otimes S \stackrel{\mathrm{id} \otimes \eta}{\longrightarrow} A^{\star} \otimes A \otimes A^{\star} \stackrel{\epsilon \otimes \mathrm{id}}{\longrightarrow} S \otimes A^{\star} \cong A^{\star}
\end{aligned}
$$

are identity maps.

The map $\eta$ is the coevaluation and $\epsilon$ is the evaluation. We say that $\left(A, A^{\star}\right)$ is a dual pair. 
A module over a commutative ring is dualizable if and only if it is finitely generated and projective. A chain complex of modules over a commutative ring is dualizable if and only if it is finitely generated and projective in each degree and only finitely many degrees are nontrivial.

We say a space is dualizable if its suspension spectrum is dualizable in the stable homotopy category.

Proposition 1.2. [14, III.4.1, III.5.1] If $M$ is a closed smooth manifold then $M_{+}:=M \amalg *$ is dualizable. If $T \nu_{M}$ is the Thom space of the normal bundle of an embedding of $M$ in $\mathbb{R}^{p}$, the dual of $M_{+}$is $\Sigma^{-p} T \nu_{M}$

If $A$ is dualizable with dual $A^{\star}$ and $f: A \rightarrow A$ is a morphism in $\mathscr{V}$, the dual of $f$, denoted $f^{\star}$, is the composite

$$
A^{\star} \cong A^{\star} \otimes S \stackrel{\text { id } \otimes \eta}{\longrightarrow} A^{\star} \otimes A \otimes A^{\star} \stackrel{\text { id } \otimes f \otimes \text { id }}{\longrightarrow} A^{\star} \otimes A \otimes A^{\star} \stackrel{\epsilon \otimes \mathrm{id}}{\longrightarrow} S \otimes A^{\star} \cong A^{\star}
$$

Definition 1.3. If $A$ is dualizable with dual $A^{\star}, P, Q$ are objects of $\mathscr{V}$ and $f: P \otimes$ $A \rightarrow A \otimes Q$ is an morphism in $\mathscr{V}$, the trace of $f, \operatorname{tr}(f)$, is the composite

$$
\begin{aligned}
P \cong P \otimes S \stackrel{\mathrm{id} \otimes \eta}{\longrightarrow} P \otimes A \otimes A^{\star} \stackrel{f \otimes \mathrm{id}}{\longrightarrow} A \otimes & Q \otimes A^{\star} \\
& \downarrow \gamma \\
A^{\star} \otimes A \otimes Q & \stackrel{\epsilon \otimes \mathrm{id}}{\longrightarrow} S \otimes Q \cong Q .
\end{aligned}
$$

The trace of a linear transformation is the usual linear algebra trace. The trace of a chain map is the alternating sum of the levelwise traces. If $f$ is an endomorphism of a closed smooth manifold and $H_{*}(-: \mathbb{Q})$ is the rational homology functor, the trace of $H_{*}(f: \mathbb{Q})$ is the Lefschetz number of $f$. The trace of $f$ in the stable homotopy category is the fixed point index of $f$.

It is important to note that the trace is independent of the choice of dual, coevaluation, and evaluation.

Proposition 1.4. Let $F: \mathscr{V} \rightarrow \mathscr{W}$ be a strong symmetric monoidal functor and $A \in \mathscr{V}$ be dualizable with dual $A^{\star}$. Then $F(A)$ is dualizable with dual $F\left(A^{\star}\right)$.

For any map $f: Q \otimes A \rightarrow A \otimes P$, we have

$$
F(\operatorname{tr}(f))=\operatorname{tr}(F(Q) \otimes F(A) \rightarrow F(Q \otimes A) \stackrel{F(f)}{\longrightarrow} F(A \otimes P) \rightarrow F(A) \otimes F(P)) .
$$

The rational homology functor is a strong symmetric monoidal functor and for each stable map $M \rightarrow N$ there is an induced map $H_{*}(M ; \mathbb{Q}) \rightarrow H_{*}(N, \mathbb{Q})$. In particular, if $\pi_{0}^{s}\left(S^{0}\right)$ is the zeroth stable homotopy group of $S^{0}$, there is a map $\iota: \pi_{0}^{s}\left(S^{0}\right) \rightarrow \operatorname{Hom}(\mathbb{Q}, \mathbb{Q})$. The map $\iota$ is injective and there is an isomorphism $\mathbb{Z} \rightarrow \pi_{0}^{s}\left(S^{0}\right)$ where the image of $1 \in \mathbb{Z}$ under $\iota$ is the identity map of $\mathbb{Q}$.

Corollary 1.5. [3] If $f: M \rightarrow M$ is an endomorphism of a closed smooth manifold the image of the fixed point index of $f$ under the injection $\iota$ is the Lefschetz number of $f$.

There are two results about the trace that will be used to compare the invariants defined here with previously defined approaches to coincidence invariants. Both are easily verified using string diagrams [8,17].

Lemma 1.6. Given dualizable objects $A$ and $B$ and isomorphisms $\psi_{A}: A^{\star} \rightarrow A \otimes P$ and $\psi_{B}: Q \otimes B \rightarrow B^{\star}$ the trace of

$$
Q \otimes A \stackrel{1 \otimes f}{\longrightarrow} Q \otimes B \stackrel{\psi_{B}}{\longrightarrow} B^{\star} \stackrel{g^{\star}}{\longrightarrow} A^{\star} \stackrel{\psi_{A}}{\longrightarrow} A \otimes P
$$




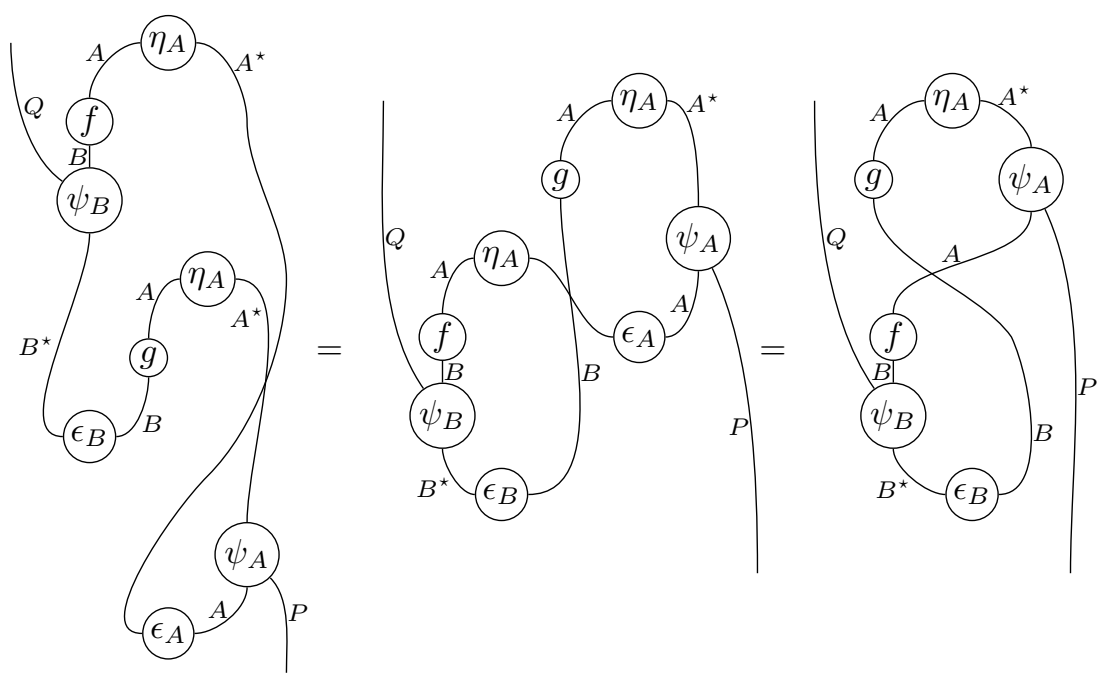

Figure 1. Proof of Lemma 1.6

is the composite

$$
\begin{gathered}
Q \simeq Q \otimes S \stackrel{1 \otimes \eta_{A}}{\longrightarrow} Q \otimes A \otimes A^{\star} \stackrel{1^{2} \otimes \psi_{A}}{\longrightarrow} Q \otimes A \otimes A \otimes P \stackrel{1 \otimes g \otimes f \otimes 1}{\longrightarrow} \\
Q \otimes B \otimes B \otimes P \stackrel{1 \otimes \gamma \otimes 1}{\longrightarrow} Q \otimes B \otimes B \otimes P \stackrel{\psi_{B} \otimes 1^{2}}{\longrightarrow} B^{\star} \otimes B \otimes P \stackrel{\epsilon_{B} \otimes 1}{\longrightarrow} S \otimes P \simeq P
\end{gathered}
$$

Suppose given dualizable objects $A$ and $B$ in a symmetric monoidal category along with maps $\phi_{A}: A \rightarrow A \otimes A$ and $\hat{\beta}: Q \otimes B \rightarrow B^{\star}$. For maps $g: A \rightarrow B$ and $\alpha: S \rightarrow A$ define $g^{\phi, \alpha}: B \rightarrow A$ to be the composite

$Q \otimes B \stackrel{\hat{\beta}}{\rightarrow} B^{\star} \stackrel{g^{\star}}{\longrightarrow} A^{\star} \simeq A^{\star} \otimes S \stackrel{1 \otimes \alpha}{\longrightarrow} A^{\star} \otimes A \stackrel{1 \otimes \phi_{A}}{\longrightarrow} A^{\star} \otimes A \otimes A \stackrel{\epsilon_{A} \otimes 1}{\longrightarrow} S \otimes A \simeq A$.

Let $\beta: Q \otimes B \otimes B \rightarrow S$ be the composite $Q \otimes B \otimes B \stackrel{1 \otimes \gamma}{\longrightarrow} Q \otimes B \otimes B \stackrel{\hat{\beta} \otimes 1}{\longrightarrow} B^{\star} \otimes B \stackrel{\epsilon_{B}}{\longrightarrow}$ $S$.

Lemma 1.7. The trace of $f \circ g^{\phi, \alpha}$ is the composite

$$
Q \stackrel{1 \otimes \alpha}{\longrightarrow} Q \otimes A \stackrel{1 \otimes \phi_{A}}{\longrightarrow} Q \otimes A \otimes A \stackrel{1 \otimes g \otimes f}{\longrightarrow} Q \otimes B \otimes B \stackrel{\beta}{\rightarrow} S .
$$

\section{LeFsChetZ Numbers}

Following [4, 6, $9,12,20,21$ we start from the observation that the coincidence points of maps $f, g: M \rightarrow N$ are the intersection of the diagonal in $N$ with the image of the product

$$
f \times g: M \rightarrow N \times N \text {. }
$$

If we use $\nu \triangle \subset N \times N$ to denote the normal bundle of the diagonal in $N \times N$ and $T \nu_{\triangle \subset N \times N}$ to denote the Thom space of this bundle, for coincidence free maps the composite

$$
M \stackrel{f \times g}{\longrightarrow} N \times N \longrightarrow T \nu \triangle \subset N \times N
$$




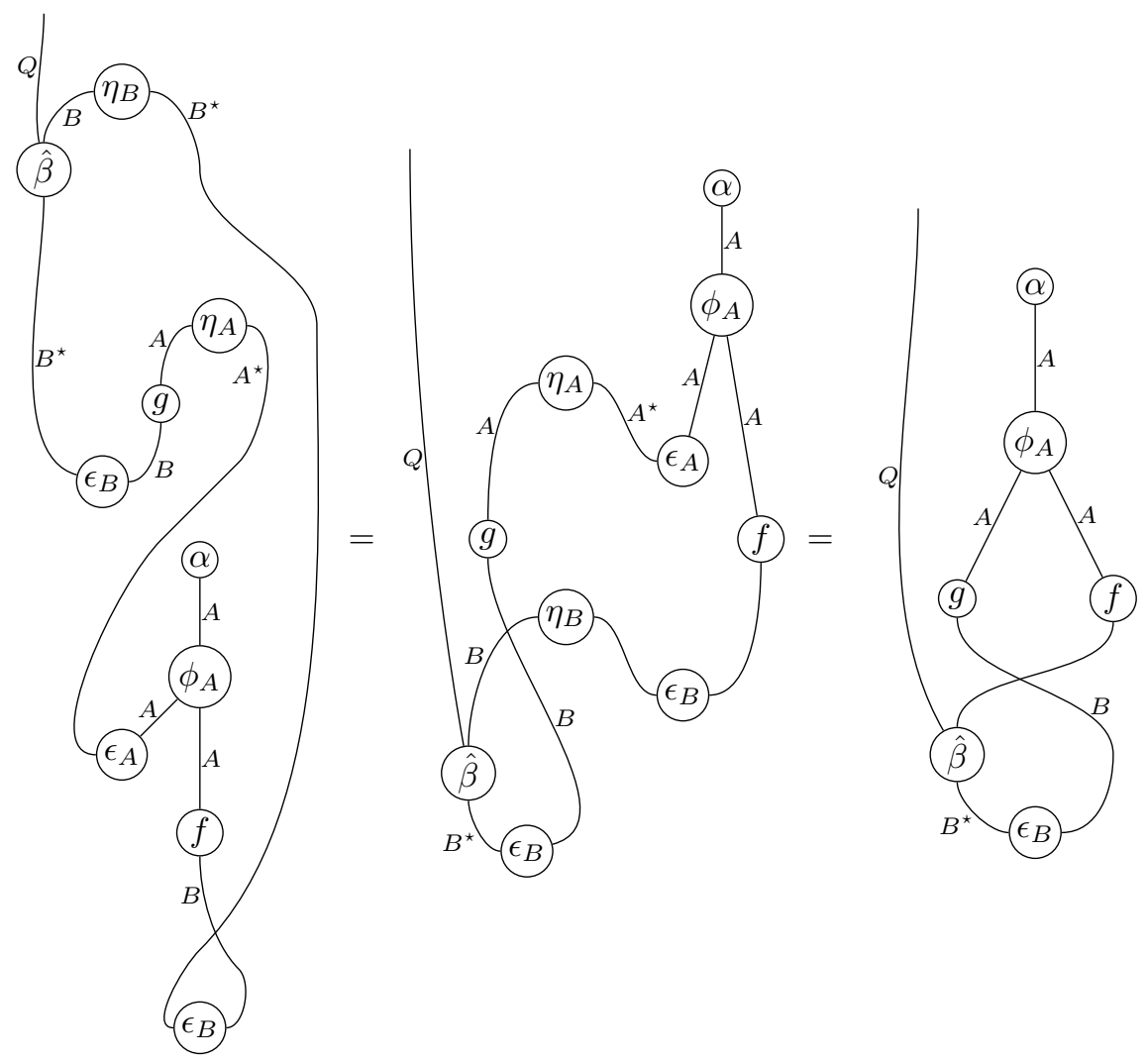

Figure 2. Proof of Lemma 1.7

where the second map is the Thom collapse will be homotopic to the constant map at the collapse point. We denote this composite by $f \times g$ since context will make the meaning unambiguous.

To define the invariants described in the introduction and prove comparison results we need some additional structure. In this paper we encode that structure using a stable map

$$
\theta: K \wedge T \nu_{\triangle \subset N \times N} \rightarrow M_{+} \wedge L
$$

This is exactly the structure needed to define traces as in the previous section and apply Proposition 1.4. If $f$ and $g$ have no coincidences the composite

$$
K \wedge M_{+} \stackrel{\operatorname{id}_{K} \wedge(f \times g)}{\longrightarrow} K \wedge T \nu \triangle \subset N \times N \stackrel{\theta}{\longrightarrow} M_{+} \wedge L
$$

will be homotopically trivial.

Definition 2.1. The coincidence index of $f$ and $g$ relative to $\theta$ is the symmetric monoidal trace of the composite

$$
K \wedge M_{+} \stackrel{\operatorname{id}_{K} \wedge(f \times g)}{\longrightarrow} K \wedge T \nu \triangle \subset N \times N \stackrel{\theta}{\longrightarrow} M_{+} \wedge L
$$

The homotopy class of the index is clearly trivial if $f$ and $g$ have no coincidences or are homotopic to maps without coincidences. 
There is also a corresponding Lefschetz number. It is the symmetric monoidal trace of the composite

$$
H_{*}(K) \otimes H_{*}\left(M_{+}\right) \stackrel{\operatorname{id} \otimes(f \times g)_{*}}{\longrightarrow} H_{*}(K) \otimes H_{*}(T \nu \triangle \subset N \times N) \stackrel{\theta_{*}}{\longrightarrow} H_{*}(L) \otimes H_{*}\left(M_{+}\right)
$$

where $H_{*}(-)$ is rational homology or any other homology theory with a Künneth isomorphism.

Proposition 1.4 implies the following result.

Theorem B. The map induced on homology by the coincidence index of $f$ and $g$ relative to $\theta$ is the same as the Lefschetz number of $H_{*}(f)$ and $H_{*}(g)$ relative to $H_{*}(\theta)$.

This theorem is the coincidence generalization of the familiar Lefschetz-Hopf result that compares topologically and algebraically defined fixed point invariants. As the index is zero for any pair of maps that are homotopic to coincidence free maps, this implies the usual statement that the Lefschetz number of coincidence free maps is zero.

To demonstrate the value of this approach we now provide two examples of the required comparison map $\theta$. These maps will allow us to recover Theorem $\mathrm{A}$ as well as generalizations in [20,21].

2.1. Orientability. Let $k_{*}$ be a homology theory and suppose $M$ and $N$ are $k_{*}$ orientable. If $k$ is the spectrum associated to $k_{*}$ there are Thom isomorphisms [15, 20.5.8]

$$
\psi_{M}: k \wedge T \nu_{M} \cong k \wedge \Sigma^{p-m} M_{+} \quad \text { and } \quad \psi_{N}: k \wedge T \nu_{\triangle} \subset N \times N \cong k \wedge \Sigma^{n} N_{+}
$$

where $\nu_{M}$ is the normal bundle of an embedding of $M$ in $\mathbb{R}^{p}, m$ is the dimension of $M$, and $n$ is the dimension of $N$.

The Thom isomorphism induce the familiar homology isomorphisms

$\tilde{k}_{i}\left(T \nu_{M}\right) \cong \tilde{k}_{i}\left(\Sigma^{p-m} M_{+}\right) \cong \tilde{k}_{i-p+m}\left(M_{+}\right) \quad \tilde{k}_{i}(T \nu \triangle \subset N \times N) \cong \tilde{k}_{i}\left(\Sigma^{n} N_{+}\right) \cong \tilde{k}_{i-n}\left(N_{+}\right)$ and define a map

$$
\begin{array}{rr}
\theta: k \wedge S^{p-m} \wedge T \nu \triangle \subset N \times N & k \wedge S^{p-2 m+n} \wedge M_{+} \\
\downarrow 1 \wedge \psi_{N} & 1 \wedge \psi_{M} \uparrow \\
k \wedge S^{p-m+n} \wedge N_{+} \longrightarrow & \pi
\end{array}
$$

where $\pi$ is the projection map for $N$ and $C$ is the Thom collapse for an embedding of $M$ in $\mathbb{R}^{p}$.

Theorem 2.2. If $k_{*}$ has a Künneth isomorphism and $M$ and $N$ are closed smooth $k$-orientable manifolds the stable homotopy class of the index with respect to this $\theta$ is the same as

$$
\sum_{i}(-1)^{i} \operatorname{tr}\left(\begin{array}{cc}
\tilde{k}_{i}\left(M_{+}\right) \stackrel{f_{*}}{\longrightarrow} \tilde{k}_{i}\left(N_{+}\right) & \tilde{k}_{m-n}\left(S^{0}\right) \otimes \tilde{k}_{i}\left(M_{+}\right) \\
\tilde{k}_{i+q-n}\left(T \nu_{N}\right) \stackrel{\left(g^{\star}\right)_{*}}{\longrightarrow} \tilde{k}_{i+p-n}\left(T \nu_{M}\right)
\end{array}\right) .
$$

Since Poincaré duality is the composite of Spanier-Whitehead duality and the Thom isomorphism, this sum of traces agrees with the sum in Theorem A. As the index of coincidence free maps is trivial this theorem implies Theorem A. 
Proof. The index of $f$ and $g$ is the symmetric monoidal trace of the composite

$$
k \wedge S^{m} \wedge M_{+} \stackrel{\operatorname{id} \wedge \operatorname{id} \wedge(f \times g)}{\longrightarrow} k \wedge S^{m} \wedge T \nu \triangle \subset N \times N \stackrel{\theta}{\longrightarrow} k \wedge S^{n} \wedge M_{+} .
$$

This agrees with the trace of the composite

$$
\tilde{k}_{*}\left(S^{m} \wedge M_{+}\right) \stackrel{k_{*}(f \times g)}{\longrightarrow} \tilde{k}_{*}\left(T \nu_{\triangle \subset N \times N)} \stackrel{k_{*}(\theta)}{\longrightarrow} \tilde{k}_{*}\left(S^{n} \wedge M_{+}\right) .\right.
$$

The trace is invariant under cyclic permutation and the trace is the identity functor on endomorphisms of the unit object [17, so the trace of $k_{*}(\theta) k_{*}(f \times g)$ is the same as the composite

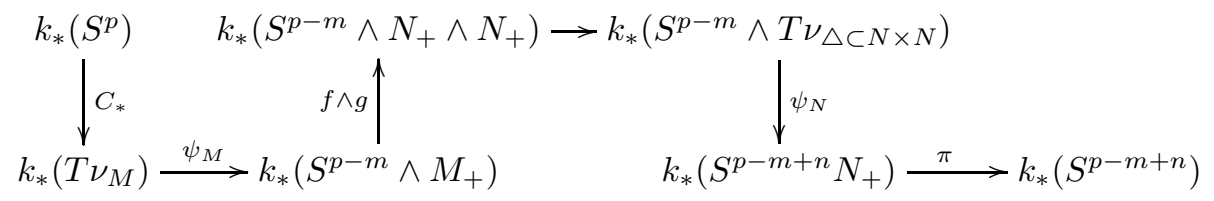

The Thom isomorphisms are compatible with the evaluation map for $N$ and the coevaluation map for $M$ in the sense that the diagrams
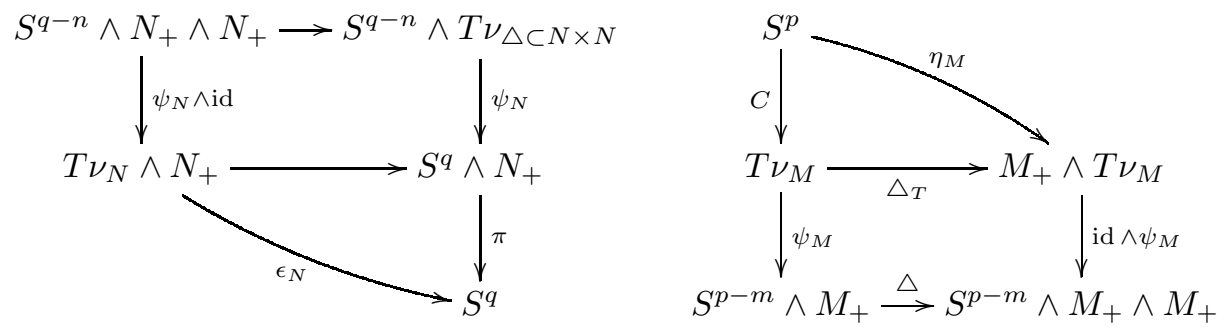

commute. ( $k$ 's have been omitted for readability.) This allows us to rewrite the composite above as

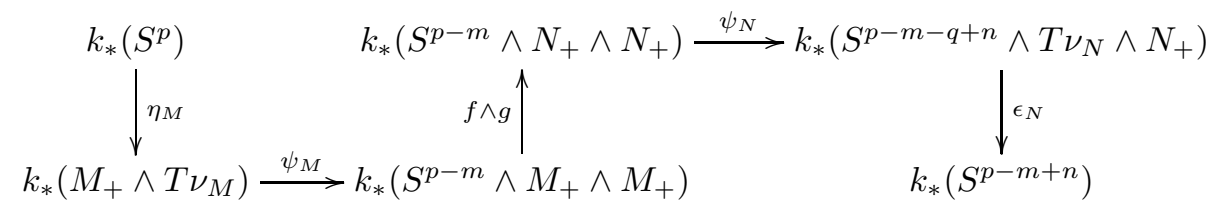

Then Lemma 1.6 implies this composite agrees with the description given in the statement of the theorem.

2.2. (Co)Homology Classes. This proof suggests a further generalization where we replace the fundamental classes by arbitrary homology and cohomology classes. Let $k$ be a multiplicative cohomology theory with multiplication $\mu$.

Proposition 2.3. For classes $\alpha \in k_{a}\left(M_{+}\right)$and $\beta \in k^{b}\left(T \nu_{\triangle \subset N \times N}\right)$ the map

$$
k_{*}\left(S^{a}\right) \stackrel{\alpha}{\longrightarrow} k_{*}\left(M_{+}\right) \stackrel{(f \times g)_{*}}{\longrightarrow} k_{*}(T \nu \triangle \subset N \times N) \stackrel{\beta(-)}{\longrightarrow} k_{*}\left(S^{b}\right)
$$


is the trace of the composite

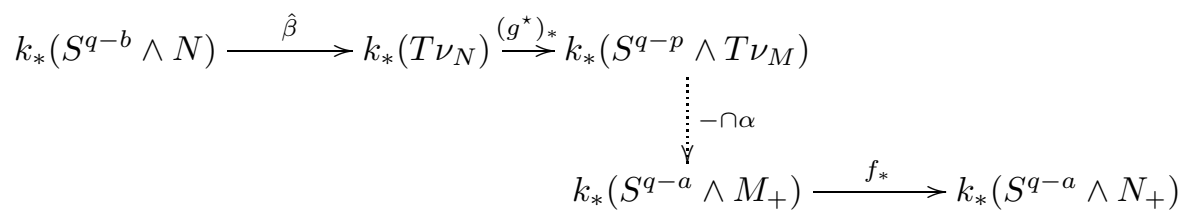

The map $\hat{\beta}$ is as in Lemma 1.7. We abuse notation and let $-\cap \alpha$ applied to $\delta \in k_{c}\left(T \nu_{M}\right)$ be the composite along the top and right sides of the diagram below.

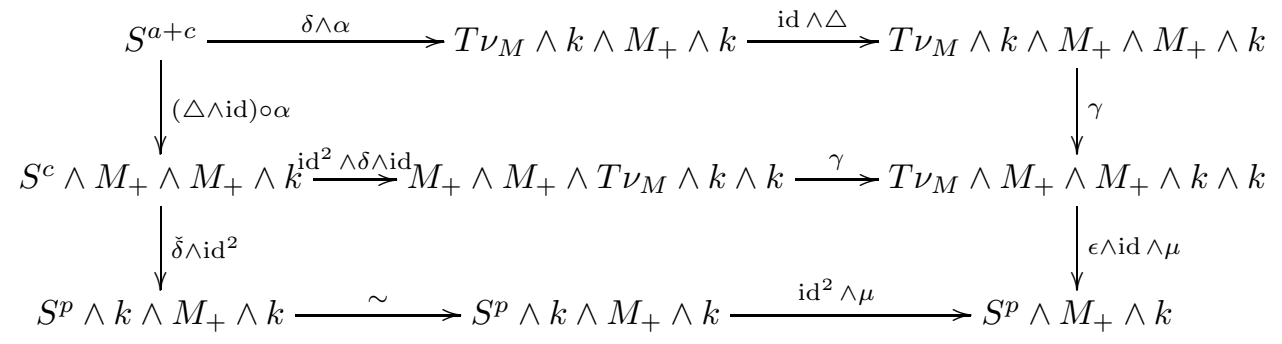

We let $\check{\delta}$ be the composite $M_{+} \wedge S^{c} \stackrel{\text { id } \wedge \delta}{\longrightarrow} M_{+} \wedge T \nu_{M} \wedge k \simeq T \nu_{M} \wedge M_{+} \wedge k \stackrel{\epsilon \wedge \text { id }}{\longrightarrow} S^{p} \wedge k$ and observe that the image of $\delta$ under $-\cap \alpha$ is the usual cap product of $\alpha$ and $\check{\delta}$.

Proof. By Lemma 1.7, the trace of the composite

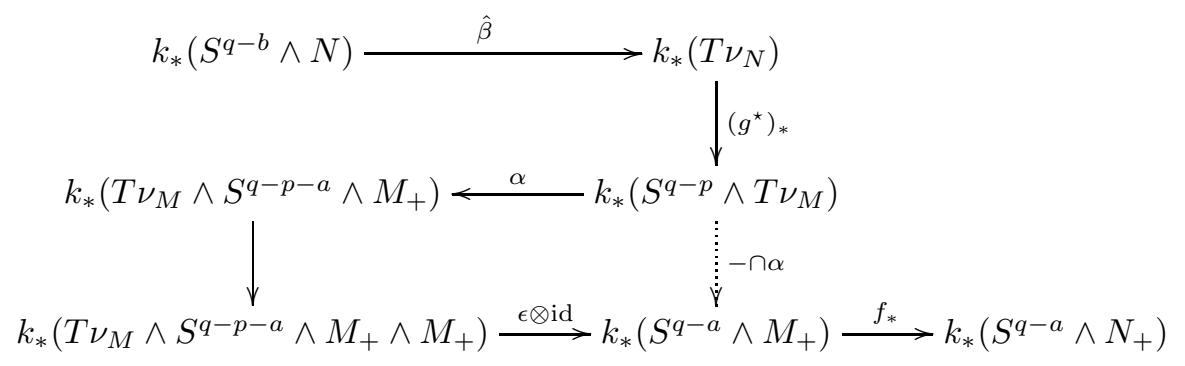

is the same as $k_{*}\left(S^{a}\right) \stackrel{\alpha}{\longrightarrow} k_{*}\left(M_{+}\right) \stackrel{(f \times g)_{*}}{\longrightarrow} k_{*}(T \nu \triangle \subset N \times N) \stackrel{\beta(-)}{\longrightarrow} k_{*}\left(S^{b}\right)$.

Classes $\alpha \in k_{a}\left(M_{+}\right)$and $\beta \in k^{b}\left(T \nu_{\triangle \subset N \times N}\right)$ are associated to stable maps $\alpha: S^{a} \rightarrow M_{+} \wedge k$ and $\beta: T \nu_{\triangle \subset N \times N} \wedge S^{-b} \rightarrow k$ and using these descriptions the map in Proposition 2.3 can also be written as the composite

$$
S^{a} \stackrel{\alpha}{\longrightarrow} M_{+} \wedge k \stackrel{f \times g}{\longrightarrow} T \nu_{\triangle \subset N \times N} \wedge k \stackrel{\beta}{\longrightarrow} S^{b} \wedge k \wedge k \stackrel{\text { id } \wedge \mu}{\longrightarrow} S^{b} \wedge k .
$$

To compare with [20,21] we restrict to the case of rational homology. There is a composite

$$
\begin{aligned}
& \operatorname{Hom}\left(H_{*}(N), H_{*+i}(N)\right) \\
& \uparrow \sim \\
& \operatorname{Hom}\left(H^{*}(N), \mathbb{Q}\right) \otimes H_{*+i}(N) \longrightarrow H^{*}(N) \otimes H_{*+i}(N) \stackrel{-\cap-}{\longrightarrow} H_{i}(N)
\end{aligned}
$$


where the unlabeled map is the universal coefficients isomorphism. For $\alpha \in H_{a}\left(M_{+}\right)$ the Lefschetz homomorphism of $f$ and $g$ evaluated at $\alpha$ [21, 5.1] is the image of $(2.5)$

$$
H_{*-q+n}(N) \cong H_{*}\left(T \nu_{N}\right) \stackrel{g^{*}}{\longrightarrow} H_{*-q+p}\left(T \nu_{M}\right) \stackrel{-\cap \alpha}{\longrightarrow} H_{*-q+a}(M) \stackrel{f_{*}}{\longrightarrow} H_{*-q+a}(N)
$$

under 2.4

The coincidence index of $f$ and $g$ [21, 4.2] is defined to be the composite

$$
H_{*}\left(M_{+}\right) \stackrel{f \times g}{\longrightarrow} H_{*}(T \nu \triangle \subset N \times N) \cong H_{*-n}\left(N_{+}\right)
$$

where the second map is the Thom isomorphism.

Theorem 2.6. [20, 2.3] [21] The Lefschetz homomorphism at $\alpha$ agrees with the coincidence index at $\alpha$.

Proof. We can determine the resulting element of $H_{*}(N)$ under either the Lefschetz number or coincidence index by evaluating at each element in $H^{*}(N)$.

For the Lefschetz homomorphism, first observe that for each $\iota \in H^{a-n}(N)$ the image of a homomorphism under

$$
\begin{aligned}
& \operatorname{Hom}\left(H_{*-q+n}(N), H_{*-q+a}(N)\right) \\
& \sim \uparrow \\
& H^{*-q+n}(N) \otimes H_{*-q+a}(N) \stackrel{-\cap-}{\longrightarrow} H_{a-n}(N) \stackrel{\iota(-)}{\longrightarrow} \mathbb{Q}
\end{aligned}
$$

is the same as the image under

$$
\begin{aligned}
& \operatorname{Hom}\left(H_{*-q+n}(N), H_{*-q+a}(N)\right) \\
& \sim \uparrow \\
& H^{*-q+n}(N) \otimes H_{*-q+a}(N) \stackrel{\operatorname{id} \otimes(\iota \cap-)}{\longrightarrow} H^{*-q+n}(N) \otimes H_{*-q+n}(N) \stackrel{e v}{\longrightarrow} \mathbb{Q}
\end{aligned}
$$

Using universal coefficients we can replace $e v$ with the evaluation map for $H_{*}(N)$ and then the image of a map $\psi: H_{*-q+n}(N) \rightarrow H_{*-q+a}(N)$ under 2.7 is the symmetric monoidal trace of

$$
H_{*-q+n}(N) \stackrel{\psi}{\longrightarrow} H_{*-q+a}(N) \stackrel{\iota \cap-}{\longrightarrow} H_{*-q+n}(N) .
$$

Then the Lefschetz homomorphism evaluated at $\alpha$ and $\iota$ is the trace of

$H_{*-q+n}(N) \cong H_{*}\left(T \nu_{N}\right) \stackrel{\left(g^{\star}\right)_{*}}{\longrightarrow} H_{*-q+p}\left(T \nu_{M}\right) \stackrel{-\cap\left(f^{*}(\iota) \cap \alpha\right)}{\longrightarrow} H_{*-q+n}(M) \stackrel{f_{*}}{\longrightarrow} H_{*-q+n}(N)$.

Applying Proposition 2.3 with $f^{*}(\iota) \cap \alpha$ as the homology class for $M_{+}$we see that the Lefschetz homomorphism evaluated at $\alpha$ and $\iota$ is the composite

$$
H_{*}\left(S^{n-p}\right) \stackrel{f^{*}(\iota) \cap \alpha}{\longrightarrow} H_{*}\left(M_{+}\right) \stackrel{(f \times g)_{*}}{\longrightarrow} H_{*}(T \nu \triangle \subset N \times N) \stackrel{\beta(-)}{\longrightarrow} H_{*}\left(S^{n}\right)
$$

where $\beta$ is the trivialization of the tangent bundle in rational homology followed by the projection. In particular, the diagram

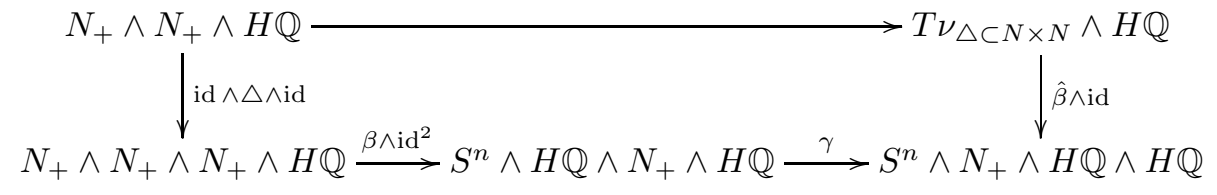

where the unlabeled horizontal map is a collapse and $\hat{\beta}$ is the Thom isomorphism commutes. Then Figure 3 recovers the form of the coincidence index in 21]. 


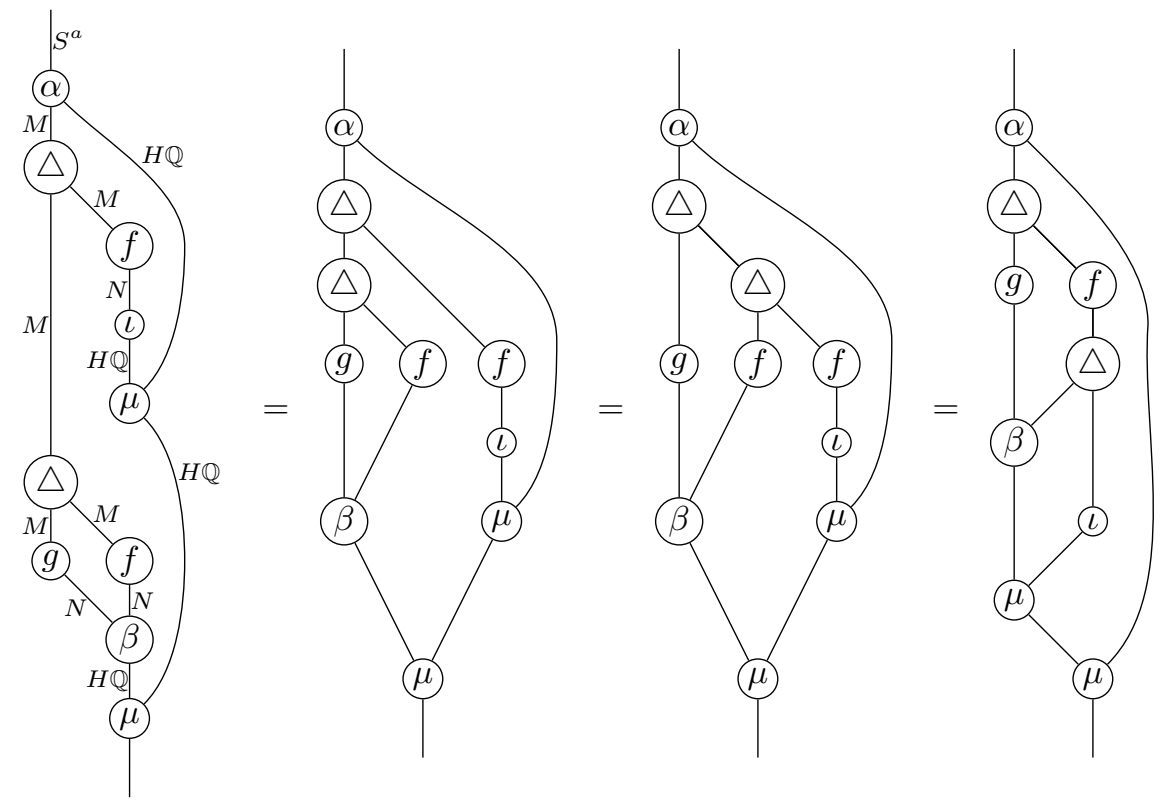

FIgURE 3. Comparison with the coincidence index in [21].

2.3. Intersections. These results are not specific to coincidences but apply to all intersections. As the generalizations are straightforward we only sketch the ideas.

Let $Q$ be a submanifold of a manifold $P$ and $f: M \rightarrow P$ be a continuous map. If the image of $f$ is disjoint from $Q$ the composite of $f$ with the Thom collapse for the normal bundle of $Q$ in $P$

$$
M \stackrel{f}{\longrightarrow} P \longrightarrow T \nu_{Q \subset P}
$$

is trivial. It is homotopically trivial if $f$ is homotopic to a map $g$ whose image is disjoint from $Q$. In general the converse is not true, see 9 for a refinement that gives a necessary and sufficient condition.

As in the previous section a stable map $\theta: K \wedge T \nu_{Q \subset N} \rightarrow L \wedge M_{+}$defines both an index and Lefschetz number.

Definition 2.8. The intersection index of $f$ and $Q$ relative to $\theta$ is the symmetric monoidal trace of the composite

$$
K \wedge M_{+} \stackrel{\text { id } \wedge f}{\longrightarrow} K \wedge T \nu_{Q \subset N} \stackrel{\theta}{\longrightarrow} L \wedge M_{+} .
$$

The Lefschetz number is the symmetric monoidal trace of the composite

$$
H_{*}(K) \otimes H_{*}\left(M_{+}\right) \stackrel{\operatorname{id} \otimes f_{*}}{\longrightarrow} H_{*}(K) \otimes H_{*}\left(T \nu_{Q \subset N}\right) \stackrel{\theta_{*}}{\longrightarrow} H_{*}(L) \otimes H_{*}\left(M_{+}\right)
$$

where $H_{*}$ is rational homology.

With these definitions Theorem B and its proof generalize immediately.

Theorem 2.9. The map induced on homology by the coincidence index of $f$ and $Q$ relative to $\theta$ is the same as the Lefschetz number of $f$ and $Q$ relative to $H_{*}(\theta)$.

The other examples for coincidences generalize similarly. 


\section{TRACES FOR BICATEGORIES}

To extend to the Reidemeister trace we need to replace the trace in a symmetric monoidal category with the trace in a bicategory. This section is a brief summary of the relevant parts of [15,16, 18].

Definition 3.1. A bicategory $\mathscr{B}$ consists of

- A collection ob $\mathscr{B}$.

- Categories $\mathscr{B}(A, B)$ for each $A, B \in$ ob $\mathscr{B}$.

- Functors

$$
\begin{gathered}
\odot: \mathscr{B}(A, B) \times \mathscr{B}(B, C) \rightarrow \mathscr{B}(A, C) \\
U_{A}: * \rightarrow \mathscr{B}(A, A)
\end{gathered}
$$

for $A, B$ and $C$ in ob $\mathscr{B}$.

Here $*$ denotes the category with one object and one morphism. The functors $\odot$ are required to satisfy unit and associativity axioms up to natural isomorphisms in $\mathscr{B}(A, B)$.

The elements of ob $\mathscr{B}$ are called 0-cells. The objects of $\mathscr{B}(A, B)$ are called 1-cells. The morphisms of $\mathscr{B}(A, B)$ are called 2-cells.

The most suggestive example for the purposes of this paper is the bicategory whose objects are rings and for each pair of rings $R$ and $S$ the associated category is the category of $R-S$ bimodules and their homomorphisms. The composition is given by tensor product and a ring regarded as a module over itself is the unit.

Definition 3.2. [15, 16.4.1] A 1-cell $X \in \mathscr{B}(A, B)$ is right dualizable with dual $Y \in \mathscr{B}(B, A)$ if there are 2-cells

$$
\eta: U_{A} \longrightarrow X \odot Y \quad \epsilon: Y \odot X \longrightarrow U_{B}
$$

such that the composites

$$
\begin{aligned}
& Y \cong Y \odot U_{A} \stackrel{\mathrm{id} \odot \eta}{\longrightarrow} Y \odot X \odot Y \stackrel{\epsilon \odot \text { id }}{\longrightarrow} U_{B} \odot Y \cong Y \\
& X \cong U_{A} \odot X \stackrel{\eta \odot \mathrm{id}}{\longrightarrow} X \odot Y \odot X \stackrel{\text { id } \odot \epsilon}{\longrightarrow} X \odot U_{B} \cong X
\end{aligned}
$$

are identity maps.

The map $\eta$ is the coevaluation and $\epsilon$ is the evaluation. We say $(X, Y)$ is a dual pair.

Like the symmetric monoidal trace, the trace of a 2-cell is defined using a composite of the coevaluation and evaluation for a dual pair. Unlike that case, the source of the evaluation and target of the coevaluation are not isomorphic. To accommodate this, we need more structure on a bicategory before we can define the trace.

Definition 3.3. [16, 4.4.1] A shadow for a bicategory $\mathscr{B}$ is a functor

$$
\langle-\rangle: \coprod \mathscr{B}(A, A) \rightarrow \mathscr{T}
$$

to a category $\mathscr{T}$ and unital and associative natural isomorphisms $\langle\langle X \odot Y\rangle \cong\langle Y \odot X\rangle$ for every pair of 1-cells $X \in \mathscr{B}(A, B)$ and $Y \in \mathscr{B}(B, A)$. 
Definition 3.4. [16, 4.5] Let $X$ be a dualizable 1-cell in $\mathscr{B}$ with dual $Y$ and $f: Q \odot X \rightarrow X \odot P$ be a 2-cell in $\mathscr{B}$. The trace of $f$ is the composite

$$
\begin{aligned}
& \langle Q Q\rangle \cong\left\langle Q \odot U_{A}\right\rangle \stackrel{\text { id } \odot \eta}{\longrightarrow}\langle\langle Q \odot X \odot Y\rangle \\
& \downarrow f \odot \text { id } \\
& \left\langle\langle X \odot P \odot Y \rangle \stackrel { \sim } { \longrightarrow } \left\langle\langle P \odot Y \odot X \rangle \stackrel { \operatorname { i d } \odot \epsilon } { \longrightarrow } \left\langle\left\langle P \odot U_{B}\right\rangle \cong\langle\langle P\rangle\rangle .\right.\right.\right.
\end{aligned}
$$

The main result of the next section is a comparison of traces in two different bicategories. One of these traces is a more familiar description of the Reidemeister trace and the other is an alternative description that has some significant technical advantages. We first describe the relevant bicategories.

3.1. GpTop. The 0-cells in the bicategory GpTop are finite groups. A 1-cell $X: G \rightarrow$ $H$ is a based space with an action of $G \times H$ where the actions of $G$ and $H$ are separately free away from the base point. The morphisms from $X: G \rightarrow H$ to $Y: G \rightarrow H$ are stable homotopy classes of equivariant maps from $X$ to $Y$.

The bicategorical composition is given by the smash product followed by the quotient by the diagonal action. The unit object associated to a finite group $G$ is $G_{+}$regarded as a $G-G$ set with a trivial action on the base point. The shadow in GpTop is the quotient by the diagonal action of the group.

Theorem 3.5. [16, 3.2.3] For a closed smooth manifold $M$ the universal cover $\tilde{M}_{+}$is dualizable as $a * \times \pi_{1}(M)$ space in the bicategory GpTop.

For a continuous map $f: M \rightarrow M$ there are induced maps $f_{*}: \pi_{1}(M) \rightarrow \pi_{1}(M)$ and $\tilde{f}: \tilde{M} \rightarrow \tilde{M}$ (with some care for base points). The map $\tilde{f}$ is not $\pi_{1}(M)$ equivariant but it does induce an equivariant map $\tilde{M} \rightarrow \tilde{M} \odot\left(\pi_{1} M\right)_{f}$ where $\pi_{1}(M)_{f_{*}}$ is the $\pi_{1}(M)-\pi_{1}(M)$ set $\pi_{1}(M)$ where the right action is via $f_{*}$.

Theorem 3.6. [16, 3.2.3] The trace of $\tilde{f}: \tilde{M} \rightarrow \tilde{M} \odot\left(\pi_{1} M\right)_{f}$ is the Reidemeister trace.

3.2. Ex. A parametrized space over a space $B$ is a space $E$ along with maps $\sigma: B \rightarrow E$ and $p: E \rightarrow B$ such that $p \circ \sigma$ is the identity map of $B$. A map of parametrized spaces commutes with both $p$ and $\sigma$. For notation and terminology we will follow [19] which builds on [15].

Parametrized spaces are the 1-cells in a bicategory Ex defined in [15]. The 0-cells are topological spaces and a 1-cell from $A$ to $B$ is a parametrized space over $A \times B$. The two cells are fiberwise stable homotopy classes of maps. In the examples here the bicategory composition is given by a fiberwise smash product followed by the pullback along the diagonal map and by quotienting the resulting section. For this bicategory we will follow the notation and conventions of [19, §3].

Theorem 3.7. [15, 18.5.1, 18.6.1] If $M$ is a closed smooth manifold $S_{M}^{0}:=M \amalg M$, regarded as a parametrized space over $* \times M$, is right dualizable.

For a map of topological spaces $f: X \rightarrow Y$, we define spaces

$$
\begin{aligned}
& P(\text { id, } f):=\left\{(\gamma, x) \in Y^{I} \times X \mid \gamma(0)=f(x)\right\} \\
& P(f, \text { id }):=\left\{(x, \gamma) \in X \times Y^{I} \mid \gamma(1)=f(x)\right\}
\end{aligned}
$$

The first has a map to $Y \times X$ by $(\gamma, x) \mapsto(\gamma(1), x)$ and the second has a similar map to $X \times Y$. These become parametrized spaces with the addition of a disjoint section. We let $Y_{f}:=P(\mathrm{id}, f) \amalg(Y \times X)$ and ${ }_{f} Y:=P(f$, id $) \amalg(X \times Y)$. 
Theorem 3.8. [15, 17.3.1] For any map of spaces $f: X \rightarrow Y\left({ }_{f} Y, Y_{f}\right)$ is a dual pair.

In the bicategory Ex the shadow is given by pulling back along the diagonal map and then quotienting by the resulting section. In particular, for an endomorphism $f: X \rightarrow X,\left\langle X_{f}\right\rangle \cong\left(\Lambda^{f} X\right)_{+}$.

\section{Reidemeister trace}

Now we consider corresponding generalizations to the Nielsen number and Reidemeister trace to coincidences. There is a coincidence Nielsen number 22 but our interest here is the Reidemeister trace and so we are looking for a trace description.

A continuous map $f: M \rightarrow M$ induces a fiberwise map $S_{M} \rightarrow S_{M} \odot M_{f}$.

Theorem 4.1. The bicategorical trace of $S_{M} \rightarrow S_{M} \odot M_{f}$ is the Reidemeister trace of $f$.

There are two ways to approach Theorem 4.1. We can think of the invariants defined in 9 as the definition of the Reidemeister trace and then the identification we require can be found in [1. Alternatively, we can use a more classical description of the Reidemeister trace in terms of fixed point indices and fixed point classes and apply techniques from [16. We give a proof using the second approach here.

Proof. The universal cover $\tilde{M} \rightarrow M$ is classified by a map $\phi: M \rightarrow B \pi_{1}(M)$ and so the $\pi_{1}(M)$-space $\tilde{M}$ is equivalent to the pullback $M \times_{B \pi_{1}(M)} E \pi_{1}(M)$. Using the notation above we write this as

$$
\left.\tilde{M}_{+} \cong S_{M}^{0} \odot{ }_{\phi} B\left(\pi_{1}(M)\right) \odot \overline{\left(E \pi_{1}(M), \rho\right.}\right)_{+}
$$

where $\left.\overline{\left(E \pi_{1}(M), \rho\right.}\right)_{+}$is the parametrized space $E \pi_{1}(M) \amalg B \pi_{1}(M) \rightarrow B \pi_{1}(M)$ regarded as a space over $B \pi_{1}(M) \times *$. The space $E \pi_{1} M$ also has an action of $\pi_{1} M$ that commutes with the quotient map.

Both $S_{M}^{0}$ and ${ }_{\phi} B \pi_{1}(M)$ are dualizable. For $\left(\overline{E \pi_{1}(M), \rho}\right)_{+}$we do not have a dual pair in a bicategory, but we do have a map

$$
\left.\triangle ! S_{B \pi_{1}(M)}^{0} \rightarrow \overline{\left(E \pi_{1}(M), \rho\right.}\right)_{+} \wedge_{\pi_{1}(M)}\left(E \pi_{1}(M), \rho\right)_{+}
$$

over $B \pi_{1}(M) \times B \pi_{1}(M)$ and a $\pi_{1}(M) \times \pi_{1}(M)$-equivariant map

$$
\left.\left(E \pi_{1}(M), \rho\right)_{+} \odot \overline{\left(E \pi_{1}(M), \rho\right.}\right)_{+} \rightarrow \pi_{1}(M)_{+}
$$

which make the usual triangle diagrams for a dual pair commute. The first map is defined by lifting any path in $B \pi_{1}(M)$ to $E \pi_{1}(M)$ and then evaluating at the end points. Since we quotient by $\pi_{1}(M)$ this will be independent of choices. For the second map two points in the same fiber are taken to the group element that transforms one to the other.

If $\hat{f}: B \pi_{1}(M) \rightarrow B \pi_{1}(M)$ is the map induced by $f$, the commutative diagram

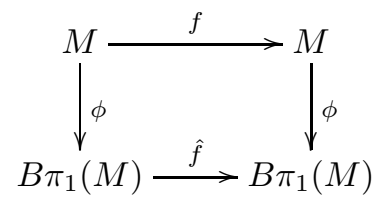


defines a map $S_{f} \odot{ }_{\phi} B \pi_{1}(M) \rightarrow{ }_{\phi} B \pi_{1}(M) \odot B \pi_{1}(M)_{B(\hat{f})}$ [19, 3.3]. We can define a map

$$
B \pi_{1}(M)_{B(\hat{f})} \odot\left(E \pi_{1}(M), \rho\right)_{+} \rightarrow\left(E \pi_{1}(M), \rho\right)_{+} \wedge_{\pi_{1}(M)} \pi_{1}(M)_{f_{*}}
$$

by $((\gamma, x), e) \mapsto \tilde{\gamma}(0)$ where $\tilde{\gamma}$ is a lift of $\gamma$ to a path ending at $\hat{f}(e)$. This is a map over $B \pi_{1}(M)$ and equivariant with respect to the right action of $\pi_{1}(M)$.

Using the identification $\tilde{M}_{+} \cong S_{M}^{0} \odot{ }_{\phi} B\left(\pi_{1}(M)\right) \odot\left(E \pi_{1}(M), \rho\right)_{+}$the composite

$$
\begin{aligned}
S_{M}^{0} \odot{ }_{\phi} B \pi_{1}(M) \odot\left(\overline{E \pi_{1}(M), \rho}\right)_{+} & \left.\stackrel{f \odot \mathrm{id}}{\longrightarrow} S_{M}^{0} \odot M_{f} \odot{ }_{\phi} B \pi_{1}(M) \odot \overline{\left(E \pi_{1}(M), \rho\right.}\right)_{+} \\
& \longrightarrow S_{M}^{0} \odot{ }_{\phi} B \pi_{1}(M) \odot B \pi_{1}(M)_{B(\hat{f})} \odot \overline{\left(E \pi_{1}(M), \rho\right)_{+}} \\
& \left.\longrightarrow S_{M}^{0} \odot{ }_{\phi} B \pi_{1}(M) \odot \overline{\left(E \pi_{1}(M), \rho\right.}\right)_{+} \wedge \pi_{1}(M)_{f_{*}} .
\end{aligned}
$$

is the map $\tilde{f}: \tilde{M} \rightarrow \tilde{M} \odot\left(\pi_{1} M\right)_{f_{*}}$ induced by $f$. Then the trace of $\tilde{f}: \tilde{M} \rightarrow$ $\tilde{M} \odot\left(\pi_{1} M\right)_{f_{*}}$ is the composite [18, 7.5] [19, 5.2]

$$
S^{0} \stackrel{\operatorname{tr}(f)}{\longrightarrow}\left\langle\left\langle M_{f}\right\rangle\right\rangle \rightarrow\left\langle\left\langle B \pi_{1}(M)_{B(\hat{f})}\right\rangle\right\rangle \rightarrow\left\langle\left\langle\pi_{1}(M)_{f_{*}}\right\rangle .\right.
$$

The composite of the second and third maps takes a twisted loop in $M$ to its associated fixed point class.

We now attempt to mimic this description for coincidences. If $Q$ is a submanifold of $P S^{\nu_{Q C P}}$ is the fiberwise one point compactification of the normal bundle of this embedding. This is a parametrized space over $Q$ where the map is induced by the projection map for the bundle. The map $Q \rightarrow S^{\nu_{Q \subset P}}$ is the section at infinity.

Corresponding to the classical Thom collapse there is a fiberwise homotopy Pontryagin-Thom collapse for $\triangle$ in $N \times N$

$$
\psi: S_{N \times N}^{0} \rightarrow S^{\nu \triangle C N \times N} \odot i_{\triangle}(N \times N)
$$

[1, §6] and [2, II.12]. Composing the fiberwise map induced by $f$ and $g S_{M}^{0} \rightarrow$ $S_{N \times N}^{0} \odot(N \times N)_{f \times g}$ with the homotopy Pontryagin-Thom collapse we have a map

$$
S_{M}^{0} \rightarrow S^{\nu \triangle C N \times N} \odot i_{\Delta}(N \times N) \odot(N \times N)_{f \times g} .
$$

Further, this is precisely the invariant that detects intersections.

Theorem 4.3. [9, Theorem 3.4] If $\operatorname{dim}(M)+3 \leq 2 \operatorname{dim}(N)$ the fiberwise stable homotopy class of 4.2 is trivial if and only if there is are maps $f^{\prime}, g^{\prime}: M \rightarrow N$, homotopic to $f$ and $g$, such that $f^{\prime}$ and $g^{\prime}$ have no coincidences.

The natural composite to consider for the coincidence Reidemeister trace is

$$
\begin{aligned}
S^{0} \rightarrow S_{M}^{0} \odot S^{\nu_{M}} \rightarrow S^{\nu \triangle C N \times N} & \odot i_{\Delta}(N \times N) \odot(N \times N)_{f \times g} \odot S^{\nu_{M}} \\
& \stackrel{?}{\rightarrow} S_{M}^{0} \odot{ }_{f} N \odot N_{g} \odot S^{\nu_{M}} \rightarrow\left\langle\left\langle\Lambda^{f, g} N\right\rangle\right.
\end{aligned}
$$

where the second to last map would need to be a generalization of the Thom isomorphisms for $M$ and $N$. To use the approach of the first section we need to rewrite the last two maps using the evaluation for the dual pair $\left(S_{M}^{0}, S^{\nu_{M}}\right)$. At this point we encounter the major difference between duality in monoidal categories and in bicategories - duality in symmetric monoidal categories is symmetric but it is sided in a bicategory. There is no adjunction that will allow us to introduce the the evaluation as we did in the previous section. This is a major obstruction to 
defining generalizations of the Reidemeister trace like those in [7] for coincidences and suggests that a very different approach may be needed.

\section{REFERENCES}

[1] M. C. Crabb. The homotopy coincidence index. J. Fixed Point Theory Appl., 7(1):1-32, 2010.

[2] Michael Crabb and Ioan James. Fibrewise homotopy theory. Springer Monographs in Mathematics. Springer-Verlag London Ltd., London, 1998.

[3] Albrecht Dold and Dieter Puppe. Duality, trace, and transfer. In Proceedings of the International Conference on Geometric Topology (Warsaw, 1978), pages 81-102, Warsaw, 1980. PWN.

[4] F. B. Fuller. The homotopy theory of coincidences. Ann. of Math. (2), 59:219-226, 1954.

[5] D. L. Gonçalves. Coincidence theory for maps from a complex into a manifold. Topology Appl., 92(1):63-77, 1999.

[6] Daciberg Gonçalves, Jerzy Jezierski, and Peter Wong. Obstruction theory and coincidences in positive codimension. Acta Math. Sin. (Engl. Ser.), 22(5):1591-1602, 2006.

[7] S. Y. Husseini. Generalized Lefschetz numbers. Trans. Amer. Math. Soc., 272(1):247-274, 1982.

[8] André Joyal and Ross Street. The geometry of tensor calculus. I. Adv. Math., 88(1):55-112, 1991.

[9] John R. Klein and E. Bruce Williams. Homotopical intersection theory. I. Geom. Topol., 11:939-977, 2007.

[10] Ulrich Koschorke. Selfcoincidences in higher codimensions. J. Reine Angew. Math., 576:1-10, 2004.

[11] Ulrich Koschorke. Geometric and homotopy theoretic methods in Nielsen coincidence theory. Fixed Point Theory Appl., (Special Issue):Art. ID 84093, 15, 2006.

[12] Ulrich Koschorke. Nielsen coincidence theory in arbitrary codimensions. J. Reine Angew. Math., 598:211-236, 2006.

[13] Solomon Lefschetz. Intersections and transformations of complexes and manifolds. Trans. Amer. Math. Soc., 28(1):1-49, 1926.

[14] L. G. Lewis, Jr., J. P. May, M. Steinberger, and J. E. McClure. Equivariant stable homotopy theory, volume 1213 of Lecture Notes in Mathematics. Springer-Verlag, Berlin, 1986. With contributions by J. E. McClure.

[15] J. P. May and J. Sigurdsson. Parametrized homotopy theory, volume 132 of Mathematical Surveys and Monographs. American Mathematical Society, Providence, RI, 2006.

[16] Kate Ponto. Fixed point theory and trace for bicategories. Astérisque, (333), 2010. arXiv:0807.1471.

[17] Kate Ponto and Michael Shulman. Duality and trace in symmetric monoidal categories. Expo. Math. 10.1016/j.exmath.2013.12.003. arXiv:1107.6032.

[18] Kate Ponto and Michael Shulman. Shadows and traces in bicategories. J. Homotopy Relat. Struct. 10.1007/s40062-012-0017-0. arXiv:0910.1306.

[19] Kate Ponto and Michael Shulman. The multiplicativity of fixed point invariants. Algebraic and Geometric Topology, 14:1275-1306, 2014. arXiv:1203.0950.

[20] Peter Saveliev. A Lefschetz-type coincidence theorem. Fund. Math., 162(1):65-89, 1999.

[21] Peter Saveliev. Lefschetz coincidence theory for maps between spaces of different dimensions. Topology Appl., 116(1):137-152, 2001. Theory of fixed points and its applications (São Paulo, 1999).

[22] Helga Schirmer. Mindestzahlen von Koinzidenzpunkten. J. Reine Angew. Math., 194:21-39, 1955. 